\title{
A obesidade e lombalgia - proposta de métodos alternativos para diminuição da massa corporal e alívio da dor: relato de caso
}

The obesity and backache - a proposal of alternative approaches for diminution of the corporal batter and relief of the pain: case report

\author{
Douglas Eduardo Contri1; Thatiana de Fátima Gomes Pereira²; Maria Raquel Lemos Canelhas ${ }^{3}$ \\ ${ }^{1}$ Fisioterapeuta, Especialista em Fisiologia do Exercício - UFSCar. \\ ${ }^{2}$ Fisioterapeuta, Especialista em Traumato-Ortopedia - Unicastelo. \\ ${ }^{3}$ Fisioterapeuta, Especialista em Fisioterapia Desportiva - Unimep. \\ Endereço para correspondência \\ Douglas Eduardo Contri \\ Av. Dr. Carlos Botelho, 3423 - Vila Nery \\ 13569-230 - São Carlos - São Paulo [Brasil] \\ douglascontri@hotmail.com
}

\begin{abstract}
Resumo
A obesidade tem sido considerada um dos mais graves problemas de saúde pública da atualidade. Neste estudo, objetivou-se correlacionar a obesidade com a dor lombar, propondo métodos alternativos para a redução da massa corporal e alívio da dor. A amostra foi composta por um indivíduo do sexo masculino, obeso e sedentário apresentando dores lombares esporadicamente. Após avaliação, o sujeito foi submetido a tratamento com alongamentos e exercícios aeróbios na bicicleta ergométrica e na esteira. O programa consistiu em 36 sessões, três vezes por semana, no período de três meses, com duração de uma hora. Foi avaliado o escore de dor lombar a cada três sessões, e o peso corporal no início e no fim do tratamento. Após análise dos resultados, concluiu-se que o programa de tratamento foi efetivo, havendo redução da massa corpórea e diminuição gradativa da dor lombar.
\end{abstract}

Descritores: Exercícios aeróbios; Dor lombar; Obesidade.

\begin{abstract}
The obesity has been considered one of the most serious problems of public health of the present time. This study it had for objective to correlate the obesity with lumbar pain, considering alternative methods for the reduction of the corporal mass and relief of pain. To research was evident with an individual of the sedentary, obese, and male sex presenting lumbar pains sporadically. After evaluation, the citizen was submitted to the treatment with allonges and aerobic exercises in the ergometric bicycle and the mat. The program consisted of 36 carried through sessions three times per week understanding three months with duration of one hour. It was evaluated the Score of lumbar Pain to each three sessions and the corporal weight in the beginning and in the end of the handling. The results showed diminution of the corporal weight and gradual diminution of the Score of Pain. It was concluded that the program of considered treatment was effective, having a reduction of the corporal mass and gradual reduction of lumbar pain.
\end{abstract}

Key words: Aerobic exercises; Obesity; Lumbar pain. 


\section{Introdução}

A obesidade tem sido considerada um dos mais graves problemas de saúde pública da atualidade $^{1}$. A prevalência do excesso de peso representa uma séria preocupação, como fator de risco para o desenvolvimento de doenças crônico-degenerativas e outras enfermidades ${ }^{2}$. Segundo estimativas da Organização Mundial da Saúde (OMS), mais de 1 bilhão de adultos apresentam excesso de peso, e desses, pelo menos, $300 \mathrm{mi}-$ lhões são obesos. No Brasil, dados da Pesquisa de Orçamentos Familiares, realizada entre 2002 e 2003 , mostraram que $40,6 \%$ da população total de adultos apresentaram sobrepeso, sendo $11 \%$ considerados obesos, com prevalência maior entre as mulheres ${ }^{3}$. Nas últimas décadas, os casos de sobrepeso e obesidade têm aumentado de forma preocupante em todo o mundo. Essa situação tem preocupado as instituições governamentais mundiais e seus diversos setores da área da saúde, uma vez que os tratamentos da obesidade e das patologias a ela relacionadas acarretam elevados custos aos sistemas de saúde pública ${ }^{4}$.

O número de pessoas com esses problemas tem alcançado índices alarmantes em muitos países industrializados. O Brasil, infelizmente, está incluído nessa síndrome do excesso de peso. Os resultados do último censo mostram que $40 \%$ dos brasileiros estão acima do peso considerado adequado. Os dados de estatísticas recentes evidenciam que, dependendo da região do Brasil, de $22 \%$ a $44 \%$ da população urbana adulta são portadores de hipertensão arterial. Esses números tornam-se extremamente importantes, pois essa doença está diretamente relacionada a eventos cerebrovasculares, coronariopatia e mortalidade. Sabe-se, ainda, que o risco desses eventos aumenta progressivamente com o aumento no nível de hipertensão arterial ${ }^{5}$.

Profissionais da área da saúde relatam que pessoas obesas correm o risco de distensão nas costas; entretanto, existem pesquisas conflitantes sobre a obesidade como "causa" ou não de dores nas costas. Pessoas obesas também podem ter dificuldade para recuperar-se de crises de dores nas costas por causa do estado geral de falta de condicionamento físico. A recuperação e a reabilitação podem levar mais tempo para elas que para pessoas em forma. Muitos médicos recomendam aos pacientes que percam o peso extra que sobrecarrega as costas ${ }^{6}$.

A dor lombar crônica pode ser causada por doenças inflamatórias, degenerativas, neoplásicas, defeitos congênitos, debilidade muscular, predisposição reumática, sinais de degeneração da coluna ou dos discos intervertebrais e outras. Entretanto, frequentemente a dor lombar crônica não decorre de doenças específicas, mas sim de um conjunto de causas, por exemplo, fatores sócio-demográficos (idade, sexo, renda e escolaridade), comportamentais (fumo e baixa atividade física), exposições ocorridas nas atividades cotidianas (trabalho físico pesado, vibração, posição viciosa, movimentos repetitivos) e outros (obesidade, morbidades psicológicas) ${ }^{7}$.

O tratamento da obesidade deve incluir modificações dietéticas, aumento na atividade física, e modificação comportamental na postura familiar e do indivíduo obeso. Acredita-se que esse tratamento deve ser baseado em uma ação multidisciplinar, integrada por profissionais, tais como professor de educação física, nutricionista, fisioterapeuta, psicólogo e médico, visando principalmente o equilíbrio biopsicossocial do indivíduo ${ }^{8}$.

Os resultados de estudos recentes mostram que o exercício físico regular reduz a massa de gordura corporal total, a subcutânea abdominal e a visceral e melhora a resistência à insulina. Além disso, sabe-se que uma única sessão dessa atividade diminui a pressão arterial em indivíduos hipertensos e que esse efeito hipotensor pode ser continuado pela repetição de sessões de exercício ao longo do tempo ${ }^{5}$.

Sendo assim, neste estudo, objetivou-se correlacionar a obesidade com a dor lombar e identificar os efeitos deletérios causados por ela, por meio da sobrecarga exercida na estrutura lombar, propondo métodos alternativos para a redução da massa corporal e alívio da dor lombar por meio de exercícios aeróbicos, resistência muscular e alongamento. 


\section{Materiais e métodos}

A amostra foi composta de um indivíduo do sexo masculino, com aproximadamente 44 anos de idade, 1,75 $\mathrm{m}$ de altura, obeso, com IMC (Índice de Massa Corpórea) de 35,9, ex-tabagista e sedentário, apresentando dores lombares esporadicamente.

Os critérios de exclusão englobaram casos de lesão ou trauma musculoesquelético, cirurgias prévias em lombar ou nas articulações de quadril, joelho e tornozelo, cardiopatia, e também se indivíduo estivesse realizando atividade física ou incluído em algum programa de emagrecimento.

Os materiais utilizados na pesquisa foram uma balança digital, bicicleta e esteira ergométricas, câmera fotográfica digital, escala comportamental $(\mathrm{EC})^{9}$ para graduação da dor na região lombar, tabela de Índice de Massa Corpórea e colchonete.

Inicialmente foi realizada uma avaliação médica para ver as condições de saúde do sujeito. Em seguida, o voluntário foi encaminhado ao nutricionista que recomendou e acompanhou um programa de reeducação alimentar para seu emagrecimento durante toda a fase da pesquisa. Logo em seguida, ele foi encaminhado à fisioterapia, e esclarecido sobre a natureza da pesquisa, estando de acordo assinou o Termo de Consentimento Livre e Esclarecido (TCLE).

Antes de iniciar o tratamento foi verificado o peso do paciente (estando somente de sunga para não sofrer alterações nos valores da pesagem), foi verificado seu Índice de Massa Corpórea (IMC), aferida a pressão arterial e aplicada a cada três sessões, antes do início do tratamento, uma escala comportamental $(\mathrm{EC})^{9}$ para graduação da dor lombar.

Antes de cada sessão, o paciente realizava alguns alongamentos básicos, sendo depois submetido a exercícios aeróbios na bicicleta ergométrica, 15 minutos, e na esteira, 30 minutos, com cargas baixas as quais foram aumentadas gradativamente em cada sessão. A pressão arterial e os batimentos cardíacos foram monitorados. O sujeito não poderia entrar em exaustão,

quando isso ocorria o exercício era interrompido. No fim de cada sessão era realizado novamente alongamento. O programa de tratamento constou de 36 sessões, três vezes por semana, com duração de uma hora, no período de três meses. Como critério de comparação dos dados, consideraram-se os valores obtidos do peso somente da $1^{\mathrm{a}}$ e da $36^{\mathrm{a}}$ sessão.

Os resultados encontrados com relação à melhora da dor lombar e perda de peso foram anotados, tabulados para melhor compreensão dos dados.

O trabalho foi aprovado pelo Comitê de Ética e Pesquisa da Unicastelo, sob protocolo número: 2073-2293/08.

\section{Resultodos}

A Figura 1 mostra os valores encontrados por meio da avaliação com a escala comportamental $(\mathrm{EC})^{9}$, para graduar a dor na região lombar do paciente, sendo aplicada a cada três sessões, antes do início do tratamento. Nota-se que houve uma diminuição gradativa dessa dor, durante as avaliações. Na primeira avaliação o paciente relatou dor no valor máximo, grau 10, já na $11^{\mathrm{a}}$ e $12^{\mathrm{a}}$ não referiu dor, grau zero.

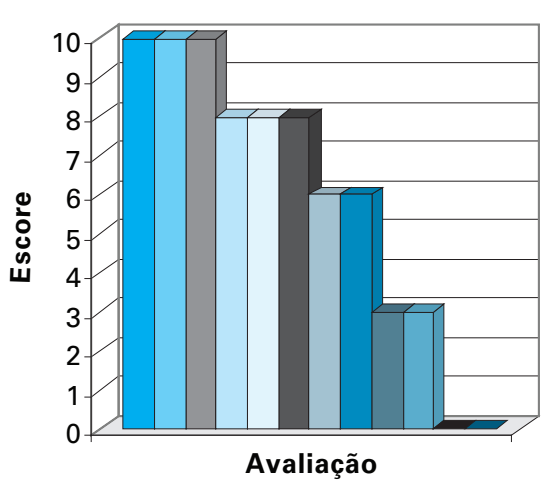

Figura l: Escore de dor apresentado pelo paciente durante as avaliações realizadas

Comparando-se o peso do paciente do início do tratamento $(110 \mathrm{~kg}$ ) com o do término (95 $\mathrm{kg}$ ), nota-se que houve uma perda de $15 \mathrm{~kg}$, e diminuição do IMC de 35,9 para 27,8 (Figura 2). 


\begin{tabular}{c|c|c}
\hline Sessão & $1^{\mathrm{a}}$ & $36^{\mathrm{a}}$ \\
\hline Peso & $110 \mathrm{~kg}$ & $95 \mathrm{~kg}$ \\
\hline IMC & 35,9 & 27,8 \\
\hline
\end{tabular}

Figura 2: Peso do paciente e IMC encontrado no início e no fim do tratamento

\section{Discussão}

A prática de atividade física regular está associada à redução de risco de desenvolvimento de diversas doenças crônicas consideradas as principais causas de morte prematura e dependência funcional de pessoas em muitos países no mundo, inclusive no Brasil. É o caso da obesidade que traz várias complicações à saúde do indivíduo. A prática da atividade física regular (6 a 7 dias na semana), em intensidade moderada, de forma contínua ou acumulada, mesmo não promovendo mudanças nos níveis de aptidão física, tem-se mostrado benéfica na redução do risco de diversas doenças ${ }^{10}$.

As demandas e o contexto da vida nas grandes cidades normalmente não propiciam oportunidades para o envolvimento do sujeito em atividades físicas, sobretudo, no período de lazer. No trabalho, exige-se menor exercício muscular e cada vez mais capacidade intelectual, por exemplo, para operar equipamentos informatizados e automatizados. O fato de a população não estar envolvida fisicamente em trabalhos ativos não traria tantas consequências se o lazer não fosse também sedentário e, o dia a dia, não estimulasse comportamento agressivo ou danoso à saúde ${ }^{11}$.

Segundo Santos ${ }^{12}$, a forma ideal de prevenir a lombalgia é recorrendo à prática de atividades físicas, que promove a diminuição da intensidade e da velocidade da implantação tanto das disfunções musculoesquelético quanto das neuromusculares e cardiorespiratórias, além de atuar positivamente nos transtornos psíquicos e no controle de massa corporal.

Para que realmente ocorra a mudança do comportamento, deve haver uma modificação na formação cultural do indivíduo e, mesmo as- sim, tal mudança será um processo lento e difícil. Parte dessa dificuldade deve-se ao fato de a sociedade atual, aceitar por normais alguns hábitos nocivos à saúde, por exemplo, o tabagismo, o uso abusivo do álcool e a alimentação inadequada, o que transforma esses comportamentos e essas pessoas em paradigmas da sociedade na atualidade sendo, muitas vezes, relacionados com os mais altos níveis de status econômico e social. Essa atitude dificulta o processo de formação de uma consciência da necessidade de um procedimento preventivo em saúde ${ }^{13}$.

Nahas ${ }^{10}$ chama a atenção para a importância da adoção de comportamentos saudáveis, evidenciando o papel coadjuvante da atividade física na busca de um estilo de vida positivo. Essa perspectiva contrasta com a realidade da manutenção de condutas de risco, uma delas é a obesidade que eleva os índices de morbidade e mortalidade.

O conceito multidisciplinar tem demonstrado a sua importância no tratamento da obesidade e da dor lombar, em detrimento do unidisciplinar, após estudos que comprovaram os resultados ${ }^{11}$. Durante um simpósio para ortopedistas, reumatologistas e fisioterapeutas, discutiu-se sobre de quem é a responsabilidade de tratar a dor lombar, e houve um consenso de que toda a equipe multidisciplinar é responsável por cuidar do doente e amenizar o sofrimento prolongado dos acometidos de dor lombar que, muitas vezes, quando não tratados precocemente, evoluem para dores crônicas ${ }^{12}$.

As pessoas com excesso de peso podem necessitar de maior esforço da coluna para sustentá-la contra a força gravitacional em razão do aumento de massa corpórea, especialmente quando se curvam para frente, levantam peso e caminham em escadas, pois ainda mais tensão é exercida nas estruturas da região lombar ${ }^{12}$.

O excesso de peso poderia ser considerado fator independente de dor lombar decorrente do aumento da circunferência abdominal, o que pode agravar essa dor e associá-la a alterações da curvatura lombar. Além disso, o aumento da carga mecânica de sustentação pela coluna seria 
o principal fator inicial dos processos degenerativos da coluna lombar ${ }^{14}$.

Pesquisas têm mostrado que à obesidade pode diminuir os mecanismos de reação contra inflamação. É bem conhecido o fato de que o tecido adiposo é metabolicamente ativo na produção de substâncias que interferem no processo antiinflamatório, o que promove uma reação anti-inflamatória de menor intensidade que dificulta a recuperação tecidual das lesões na coluna ${ }^{14}$.

Em relação ao sobrepeso e a obesidade, acredita-se que o excesso de peso corpóreo leva a uma necessidade de maior esforço da coluna lombar, ocasionando um uso excessivo e uma degeneração prematura das estruturas. No entanto, há uma divergência nos estudos quanto à possível relação entre o excesso de peso e a dor lombar, o que evidencia falta de dados que possam suportar essa hipótese ${ }^{14}$.

Vários autore ${ }^{15,16}$ relatam fortes evidências quanto à eficácia do exercício, tanto terapêutico quanto recreacional, na redução da dor lombar, indicando que a prática de atividade física é capaz de modificar índices de resposta da dor, concordando com os resultados obtidos neste estudo, no qual se verificou que, por meio de protocolo de tratamento, utilizando alongamentos e exercícios aeróbios na esteira e na bicicleta ergométrica, o paciente apresentou uma diminuição do escore de dor, que no início da terapia graduava-se em 10, (dor persistente mesmo em repouso), passando a zero (dor ausente), no término.

Mirtz et al. ${ }^{17}$ realizaram pesquisa bibliográfica, entre o período de 1990 e 2004, para determinar a associação entre lombalgia e obesidade, e apesar de encontrarem diversos estudos sobre o assunto, apenas dois identificaram diretamente a lombalgia como fator de risco para dor lombar, em outros dois trabalhos não foi verificada nenhuma relação entre o índice de massa corpórea e lombalgia, a pesquisa concluiu que há controvérsias nesse assunto, sendo necessários outros dados epidemiológicos.

Caraviello et al..$^{18}$ concluiram em seu estudo que a obesidade contribuiu para a geração e perpetuação das dores nas costas, dificultando seu tratamento, ocorrendo em razão da sobrecarga exercida pelo excesso de peso sobre as articulações da coluna vertebral.

Diversos trabalhos analisaram as respostas fisiológicas relacionadas à composição corporal, utilizando-se exercícios com predominância do metabolismo aeróbio, anaeróbio ou a combinação de ambos ${ }^{19,20,21}$.

Hauser et al. ${ }^{1}$ avaliaram os efeitos de diferentes protocolos de exercícios de curta duração sobre a composição corporal, capacidade aeróbia e força muscular em 20 mulheres obesas, divididas aleatoriamente em grupos, sendo que o Grupo I realizou 4 semanas de componente de força (exercícios contra-resistência) e aeróbio (bicicleta ergométrica e/ou esteira e treinamento em circuito com exercícios contra-resistência) e o Grupo II realizou 4 semanas de componente de força (exercícios contra-resistência) e aeróbio (bicicleta ergométrica e/ ou esteira). Os resultados demonstraram que mesmo um treinamento realizado por curto período (4 semanas) pode induzir adaptações positivas no quadro funcional dessa população.

Apesar de existir farta literatura no que se refere ao tratamento da obesidade e melhora cardiopulmonar, há pouca de pesquisas relacionadas à obesidade e dor lombar.

De acordo com os resultados encontrados neste estudo, verificou-se que a perda de peso, e consequente redução do IMC, contribuiu para a melhora de dor lombar já que antes do início do tratamento o escore de dor do paciente era 10, e durante o tratamento foi diminuindo gradativamente, até chegar, no fim, a escore zero, mostrando que a dor lombar é um dos fatores relacionados com a obesidade.

\section{Conclusões}

Concluiu-se que o programa de tratamento proposto foi efetivo, houve uma redução da massa corpórea e, consequentemente, uma diminuição da dor lombar, mostrando que o excesso de peso e a lombalgia estão diretamente relacionados. 


\section{Referências}

1. Hauser C, Benetti M, Rabelo FPV. Estratégias para o emagrecimento. Rev Bras Cieneantropom. Desempenho Hum. 2004;6(1):72-81.

2. Dias R, Prestes J, Manzatto R, Ferreira CKO, Donatto FF, Foschini D, et al. Efeitos de diferentes programas de exercício nos quadros clínico e funcional de mulheres com excesso de peso. Rev. Bras. Cineantropom. Desempenho Hum. 2006;8(3):58-65.

3. Barbato KBG, Martins RCV, Rodrigues MLG, Braga JU, Francischetti EA, Genelhu V. Efeitos da redução de peso superior a $5 \%$ nos perfis hemodinâmicos, metabólicos e neuroendócrino de obesos de grau I. Arquivos Brasileiros de Cardiologia. 2006;87(1):12-21.

4. Fernandes RA, Oliveira AR, Júnior IFF. Correlação entre diferentes indicadores de adiposidade corporal e atividade física habitual em jovens do sexo masculino. Rev. Bras.Cineantropom. Desempenho Hum. 2006;8(4):32-8.

5. Nunes APOB, Rios ACS, Cunha GA, Barretto ACP, Negrão CE. Efeitos de um programa de exercício físico não-supervisionado e acompanhado a distância, via internet, sobre a pressão arterial e composição corporal em indivíduos normotensos e pré-hipertensos. Arquivos Brasileiros de Cardiologia. 2006;86(4):289-96.

6. Hochschuler S, Reznik B. Trate sua coluna sem cirurgia. São Paulo: Manole 2000;6:103-4.

7. Silva MC, Fassa AG, Valle NCJ. Dor lombar crônica em uma população adulta do Sul do Brasil: prevalência e fatores associados. Cad Saúde Pública. 2004;20(2):377-85.

8. Teixeira LR. Comparação de dois tipos de treinamento físico na composição corporal de adolescentes obesos submetidos a tratamento multidisciplinar. 2006. 81f. [dissertação de mestrado em Medicina]. São Paulo: Universidade Federal de São Paulo; 2006.

9. Wall PD, Melzack R.Textbook of pain. ChurchillLivingstone, Edinburgh, 1990.

10. Nahas MC. Atividade física, saúde e qualidade de vida. Londrina: Midiograf, 2001.

11. Cox MH, Shephard RJ, Corey P. Physical activity and alienation in the work place. Journal of Sports Medicine. 1987;27:306-15.
12. Santos CBS. Avaliação do programa escola de postura em pacientes com lombalgia crônica do hospital municipal de Rolim de Moura - RO [dissertação de mestrado]. Brasília, DF: Universidade de Brasília - Programa de Pós Graduação em Ciências da Saúde; 2008.

13. Laat E. Estudo de episódios lombálgicos e condições de trabalho em militares. [dissertação de mestrado]. Porto Alegre: Mestrado profissionalizante em Engenharia. Universidade Federal do Rio Grande do Sul. Porto Alegre; 2005.

14. Fujii LC. Incapacidade laborativa por dor lombar e sua relação com o índice de massa corpórea em empresa de comércio varejista. [dissertação de mestrado]. Rio Grande do Sul: Faculdade de Medicina da UFRGS - Programa de Pós-graduação em Epidemiologia; 2008.

15. Furlan AD, Castro AW, Chung TM, Imanura ST, Camanho GL. Escola de coluna-programa desenvolvido no instituto de ortopedia e traumatologia do HC/FMUSP. Acta Ortopéd.Bras abr/jun. 1998;6(2):55-60.

16. Kankaanpãã M, Taimela S, Airaksonem O, Hãnninen.O. The efficacy of rehabilitation in chronic low back pain. Spine. 1999;24:1034-42.

17. Mirtz TA, Greene L. Is obesity a risk factor for low back pain? An example of using the evidence to answer a clinical question. Chiropractic \& Osteopathy 2005;13(1):2.

18. Caraviello EZ, Wasserstein S, Chamlian TR, Masiero D. Avaliação da dor e função de pacientes com lombalgia tratados com um programa de escola de postura. Acta Fisiatr. 2005;12(1):11-4.

19. Andersen RE. Exercise, an active lifestyle, and obesity. The Physician and Sports Medicine. 1999;27(10)32-41.

20. Astrup A, MaCdonald IA. Sympathoadrenal system and metabolism. In Handbook of obesity.1997.

21. Ballor DL, Harvey-Berino JR, Ades PA, Cryan J, Calles-Escandon J. Contrasting effects of resistance and aerobic training on body composition and metabolism after diet-induced weight loss.

Metabolism. 1996;45(2):179- 83. 